\title{
A Comparison Study of MIMO Water Wall Model with Linear, MFNN and ESN Models
}

\author{
Un-Chul Moon ${ }^{\dagger}$, Jaewoo Lim* and Kwang Y. Lee**
}

\begin{abstract}
A water wall system is one of the most important components of a boiler in a thermal power plant, and it is a nonlinear Multi-Input and Multi-Output (MIMO) system, with 6 inputs and 3 outputs. Three models are developed and comp for the controller design, including a linear model, a multilayer feed-forward neural network (MFNN) model and an Echo State Network (ESN) model. First, the linear model is developed by linearizing a given nonlinear model and is analyzed as a function of the operating point. Second, the MFNN and the ESN are developed by using training data from the nonlinear model. The three models are validated using Matlab with nonlinear input-output data that was not used during training.
\end{abstract}

Keywords: Water wall model, Power plant modelling, Power plant identification, Linearization, Multilayer feed-forward neural network, Echo state network

\section{Introduction}

Thermal power plants supply approximately $65 \%$ of the world's electric power [1], and a water wall system consists of a collection of metal tubes in the furnace of a thermal power plant. Recirculating water in the water wall is heated by combustion energy, and its phase changes from water to steam. Therefore, it is very important to properly model, control and analyze the water wall system in order to manage the entire boiler-turbine system [2].

Several principles, including the energy balance, mass balance and thermal equilibrium, can be used to explain the dynamics of the water wall system [3]. As a result, the water wall system is modeled as a dynamic nonlinear multi-input multi-output (MIMO) system. However, the controller for a nonlinear system is usually designed using a linear model based on a particular operating point. Therefore, the degree of nonlinearity of a water wall system needs to be investigated to adequately apply a linear control system.

If the object system has a severe nonlinearity or a wide operating range, the linear model will have a limitation in its ability to describe the dynamics of the object system [4]. An alternative for this kind of nonlinear control problem involves implementing a neural network for the modeling and controller design [5]. Since it is difficult to develop a reliable mathematical model of the nonlinear dynamics in practice, the neural network is typically trained according to the measured input-output data.

$\dagger$ Corresponding Author: School of Electrical and Electronic Engineering, Chung-Ang University, Korea. (ucmoon@cau.ac.kr)

* School of Electrical and Electronic Engineering, Chung-Ang University, Korea. (jaewoo1129@naver.com)

** Dept. of Electrical and Computer Engineering, Baylor University, Waco, TX 76798, USA. (Kwang Y Lee@baylor.edu)

Received: April 25, 2015; Accepted: August 30, 2015
Therefore, a mathematical nonlinear dynamic model is not necessary for this approach.

The multilayer feedforward neural network (MFNN) has been successfully applied to model static nonlinear systems [6]. However, it requires a number of tapped delays of the output neurons as input signals for dynamic systems, resulting in an increased number of input neurons and an extensive training time. Recurrent neural networks (RNN) with feedback connections are potential approaches that better represent dynamic systems, including Elman networks [7] and diagonal recurrent neural networks (DRNN) [8]. However, training for recurrent neural networks is relatively more complex than that for MFNN [9].

Recently, eco state network (ESN), a special kind of three layer RNN, was proposed by Jaeger [10]. The basic idea is to use a large "dynamic reservoir" to supply interesting dynamics from which the desired output is combined. One of the most interesting things of ESN is that it can be trained in a one-shot fashion without repetitively passing through the training set that is usually required to train other RNN [11].

In [12], Jaeger and others used the ESN to optimize problems and demonstrated simple applications. Lin and others used ESN to predict the stock price and to suggest stock trades [13]. Ishii and others used ESN to identify the yawing acceleration of an underwater robot [14]. Dai and others used ESN to predict the harmonic current from nonlinear loads [15]. Pan and Wang proposed a model for predictive control based on recurrent neural network, where they used ESN to identify an unknown nonlinear dynamic system [16].

In this paper, three models, including a linearized model, a MFNN model and a nonlinear ESN model, are developed and compared from the point of view of controller design for a practical water wall system in a $600 \mathrm{MW}$ oil drum 
boiler-turbine system. The three models are developed and compared using Matlab on a personal computer environment. First, we introduce the nonlinear water wall model with the energy balance, mass balance and thermal equilibrium equation. Then, the linear model is developed with a Taylor series expansion. The changes in the pole and steady state gain according to the change in the operating point are introduced. To train the MFNN and the ESN, Pseudo Random Binary Noise Signal (PRBNS) inputs are applied to the nonlinear water wall model. After training the MFNN and ESN, the performance of the linear model, MFNN and ESN is evaluated, presented and discussed.

\section{A Water Wall Modeling}

\subsection{A nonlinear water wall model}

In this paper, we consider a water wall model in a $600 \mathrm{MW}$ oil drum boiler-turbine system [3]. The water wall is located between the recirculating pump and the drum in the boiler. The circulating water in the water wall is heated by combustion in the furnace, and its phase changes from water to steam, and then it is poured into the drum.

We assume that the water wall system is a nonlinear model, as used by Usoro [3]. The mass balance equation, energy equilibrium equation, and thermal equilibrium equation can be defined according to the boiler structure and physical principles. The description of the water wall system is as follows:

Mass balance equation is given by:

$$
W_{w w o}=W_{r w}+K_{w r p s}
$$

where, $W_{w w o}$ : Mass flow of water wall outlet,

$W_{r w}$ : Mass flow of recirculating water,

$K_{\text {wrps }}$ : Mass flow of recirculating pump.

According to [3], $K_{\text {wrps }}$ accounts for recirculating pump leakages and seal injection. It is small and may be neglected.

Energy equilibrium equation is given by:

$$
M_{w w m e} \times K_{s w w m} \times \frac{d}{d t}\left(T_{w w m}\right)=Q_{w w g m}-Q_{w w m w}
$$

where, $M_{\text {wwme }}$ : Effective mass of water wall metal,

$K_{\text {swwm }}$ : Specific heat of water wall metal,

$T_{w w m}:$ Temperature of water wall metal,

$Q_{w w g m}:$ Heat transfer rate of gas to metal,

$Q_{w w m w}$ : Heat transfer rate of metal to water.

$$
M_{w w m e}=K_{m w w m}+\frac{K_{v w w} \times R_{d r w} \times H_{d r w}}{K_{s w w m} \times T_{w w m}}
$$

where, $K_{m w w m}$ : Mass of water wall metal,
$K_{v w w}:$ Volume of water wall,

$R_{d r w}:$ Density of drum water,

$H_{d r w}$ : Enthalpy of drum water,

$$
Q_{w w m w}=K_{u w w m w} \times\left(T_{w w m}-T_{d r s}\right)^{3}
$$

where, $K_{u w w m w}$ : Constant with dimension of $\left[\mathrm{W} / \mathrm{K}^{3}\right]$,

$T_{d r s}:$ Temperature of drum steam,

Thermal equilibrium equation of circulating water is given by:

$$
W_{w w o} \times\left(H_{w w o}-H_{r p o}\right)=Q_{w w m w}
$$

where, $H_{r p o}$ : Enthalpy of recirculating pump outlet,

$H_{w w o}$ : Enthalpy of water wall outlet,

The following constant values are used:

$K_{m w w m}=1063000, K_{v w w}=2318.61, K_{s w w m}=0.11, K_{u w w m w}=$ 173.5205

For (1)-(5), we define the inputs, the outputs, and the states as follows,

$$
\begin{gathered}
U=\left[u_{1}, u_{2}, u_{3}, u_{4}, u_{5}, u_{6}\right]^{T} \\
=\left[W_{r w}, H_{r p o}, R_{d r w}, H_{d r w}, T_{d r s}, Q_{w w g m}\right]^{T} \\
Y=\left[y_{1}, y_{2}, y_{3}\right]=\left[W_{w w o}, H_{w w o}, T_{w w m}\right]^{T} \\
X=[x]=\left[T_{w w m}\right]
\end{gathered}
$$

Then, the nonlinear model of water wall system is represented in the following equation,

$$
\begin{gathered}
\dot{x}=\frac{u_{6}-K_{u w w m w} \times\left(x-u_{5}\right)^{3}}{\left(K_{m w w m}+\frac{K_{v w w} \times u_{3} \times u_{4}}{K_{s w w m} \times x}\right) \times K_{s w w m}} \\
y_{1}=u_{1} \\
y_{2}=u_{2}+\frac{K_{u w w m w} \times\left(x-u_{5}\right)^{3}}{u_{1}} \\
y_{3}=x
\end{gathered}
$$

From the above equations, we notice that $y_{2}$ and $y_{3}$ of the water wall system have severe nonlinearity, while $y_{1}$ is $u_{1}$, which is a simple linear output.

\subsection{Effect of operating point to linear model}

The nonlinear model, given by (9)-(12), is linearized using the first-order Taylor approximation at an operating point. We generalized the operating points as follows:

Operating point $=\left[u_{10}, u_{20}, u_{30}, u_{40}, u_{50}, u_{60}, x_{0}, y_{10}, y_{20}, y_{30}\right]$

As an example, the operating point at a power output of $400 \mathrm{MW}$ is shown in Table 1, where steady state values of 6 inputs, 3 outputs, and a state can be observed. When all 
Table 1. The operating of water wall

\begin{tabular}{c|c}
\hline Variables & Operating value \\
\hline$u_{1}\left(W_{r w}\right)$ & 4716.7199 \\
\hline$u_{2}\left(H_{r p o}\right)$ & 721.4824 \\
\hline$u_{3}\left(R_{d r w}\right)$ & 34.4693 \\
\hline$u_{4}\left(H_{d r w}\right)$ & 733.1763 \\
\hline$u_{5}\left(T_{d r s}\right)$ & 1132.6393 \\
\hline$u_{6}\left(Q_{w w g m}\right)$ & 267289.8666 \\
\hline$y_{1}\left(W_{w w o}\right)$ & 4716.7199 \\
\hline$y_{2}\left(H_{w w o}\right)$ & 778.0994 \\
\hline$y_{3}\left(=x, T_{w w m}\right)$ & 1144.1883 \\
\hline
\end{tabular}

the variables are defined as deviations from the operation values in Table 1, the linear model at $400 \mathrm{MW}$ is given as,

$$
\begin{aligned}
\Delta \dot{x}= & -8.2535 \times 10^{-12} \Delta u_{3}-3.8803 \times 10^{-13} \Delta u_{4} \\
& +0.4129 \Delta u_{5}+5.9474 \times 10^{-6} \Delta u_{6}-0.4129 \Delta x \\
\Delta y_{1}= & \Delta u_{1} \\
\Delta y_{2}= & -0.012 \Delta u_{1}+\Delta u_{2}-14.7070 \Delta u_{5}+14.7070 \Delta x \\
\Delta y_{3}= & \Delta x
\end{aligned}
$$

We notice that (13) is a simple first order system with a pole at $\mathrm{s}=-0.4129$, and the overall system is stable without an oscillation mode. The transfer function matrix, $\bar{G}(s)$, of (13)-(16) for the 6-input 3-output system is given by

$$
\begin{aligned}
& \bar{G}(s)=\left(\begin{array}{ccc}
1 & 0 & 0 \\
-0.012 & 1 \frac{-1.214 \times 10^{-10}}{s+0.4129} \\
0 & 0 \frac{-8.254 \times 10^{-12}}{s+0.4129}
\end{array}\right. \\
& \left.\begin{array}{ccc}
0 & 0 & 0 \\
\frac{-5.707 \times 10^{-12}}{s+0.4129} & \frac{-14.71 s}{s+0.4129} & \frac{8.747 \times 10^{-5}}{s+0.4129} \\
\frac{-3.88 \times 10^{-13}}{s+0.4129} & \frac{0.4129}{s+0.4129} \frac{5.947 \times 10^{-6}}{s+0.4129}
\end{array}\right)
\end{aligned}
$$

where $G_{i j}$ is a transfer function of the $i$-th output from $j$-th input. According to (13) and (17), the linear model at 400 MW is a first order system with a pole on the left half splane.

To analyze the nonlinear model, the above linearization is performed as a function of the electrical power output. Then, the linear models that are generated at each operating point are compared from the point of view of the pole and the steady-state gain. Fig. 1 shows the movement of the pole with the operating points. The pole at $100 \mathrm{MW}$ is at 0.2335 and moves to the left as the power increases. The pole at $600 \mathrm{MW}$ is at -0.6084 . This means that the transient is becoming shorter by several times as the load demand increases.

Fig. 2 shows the steady-state gain changes of $y_{2}$ and $y_{3}$ as

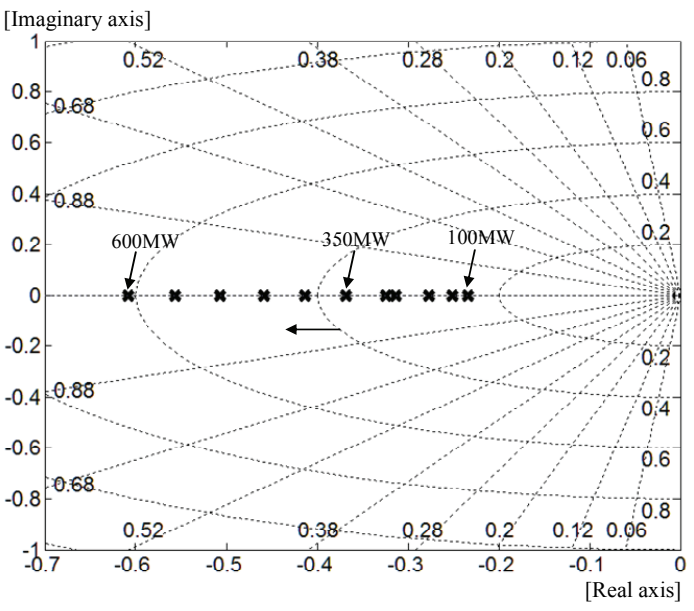

Fig. 1. Movement of the pole according to operating point
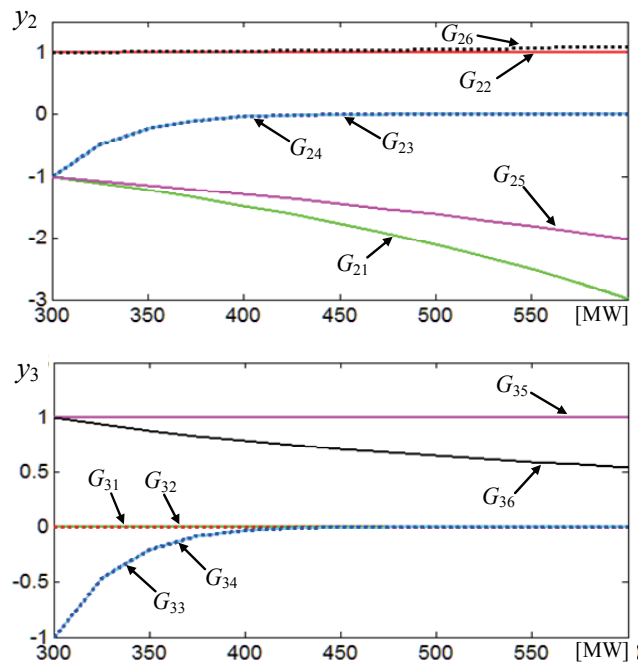

Fig. 2. The change in the steady-state gain for $y_{2}$ and $y_{3}$ with the operating point

a function of the operating points from 300 [MW] to 600 [MW], which are normalized with a gain at 300 [MW]. The gain for $y_{1}$ is not represented because it has no dynamics. In Fig. 2, the steady-state gain changes significantly. For example, the amplitude of the steady-state gain for $G_{21}$ at 600 [MW] negatively increases to three times that of 300 [MW]. From Figs. 1 and 2, even with a good nonlinear water wall model, suitable performance is hard to achieve for wide range operation using a conventional linear control technique.

\section{Two Neural Network Models}

\subsection{PRBNS training data}

As an alternative to conventional linear modeling and control, neural networks have been extensively applied to nonlinear modeling and controller design [5]. Due to its 

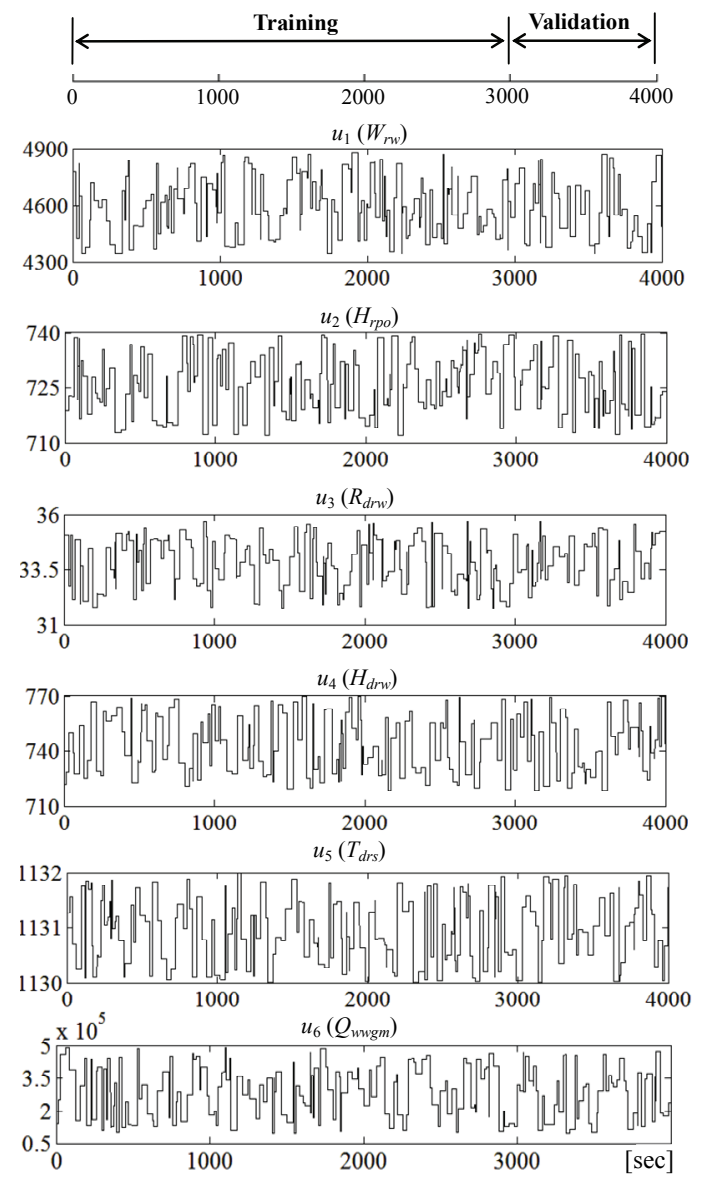

Fig. 3. PRBNS input for nonlinear water wall system

ability to learn nonlinear functions, neural networks do not need a mathematical model of the object system. Instead, the model is developed with the given input-output data. Therefore, it is very important to make a suitable selection of the training data.

To identify the dynamic system, a Pseudo Random Binary Noise Signal (PRBNS) is a popular input signal that is used to excite the system over a wide frequency range [17]. In order to obtain training data for the nonlinear water wall system considered in this paper, the PRBNS for six inputs is generated for 4000 [sec] with 0.1 [sec] sampling time.

Fig. 3 shows generated PRBNS input, and Fig. 4 shows the corresponding output using a nonlinear water wall system in (9)-(12). In these figures, the first 3000 [sec] data are used to train the neural network, and the latter 1000 [sec] data are left for validation. That is, the data between 3000 [sec] and 4000 [sec] is not used for training and is instead used to evaluate the trained neural network model.

\subsection{Multilayer feed-forward neural network model}

The multilayer feed-forward neural network (MFNN) is a standard neural network [6]. It has been extensively applied with the "error back-propagation" training algorithm
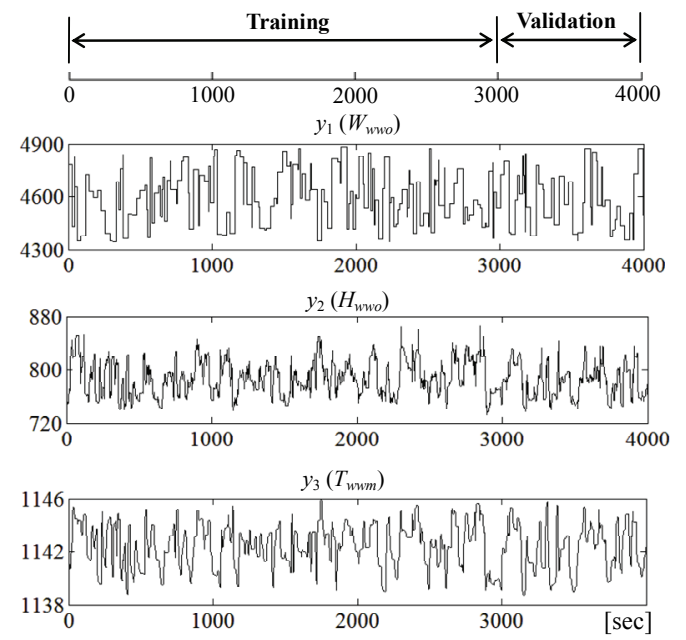

Fig. 4. Output of PRBNS input for nonlinear water wall system

to various nonlinear modeling and control problems [5].

In this paper, MFNN is first used to model a nonlinear water wall system. To train the MFNN, the training data is rearranged to reflect the water wall system of (9)-(12) as follows,

$$
Y(n+1)=f\{U(n), Y(n)\}
$$

where, $n$ is the discrete time step. And, $U(n)=\left[u_{1}(n), \cdots\right.$, $\left.u_{6}(n)\right], \quad Y(n)=\left[y_{1}(n), y_{2}(n), y_{3}(n)\right]$ and $Y(n+1)=\left[y_{1}(n+1)\right.$, $\left.y_{2}(n+1), y_{3}(n+1)\right]$. The MFNN used in this paper has a three-layer feed-forward neural network, including an input layer, a hidden layer and an output layer. The input of MFNN is $U(n)$ and $Y(n)$, and the number of the input node is 9. The output of the MFNN is $Y(n+1)$, the number of output node is 3 .

The purpose of the training is to minimize the total square error between the training output data and the MFNN output data. This is actually an unconstraint optimal problem where the error function is defined as follows,

$$
\min _{W} E_{M F N N}=\sum_{n}^{N}\left(Y_{M F N N}(n)-Y(n)\right)^{2}
$$

where $W$ represents all the weights. $Y_{M F N N}$ represents the output from the MFNN, $Y$ represents the output for the training data, $N$ represents the number for the training data, which is 30,000 and $n$ is the discrete time step. To consider the different range of each output, each output is normalized in Eq. (19). The optimal problem, (19) is solved using the back propagation algorithm [18]. The Matlab toolbox is used to train the MFNN in this paper.

One of the most important parameters for the MFNN is the number of hidden neurons. Usually, a larger number of hidden neurons decreases the error but increases the training time of the MFNN. Therefore, some kind of 


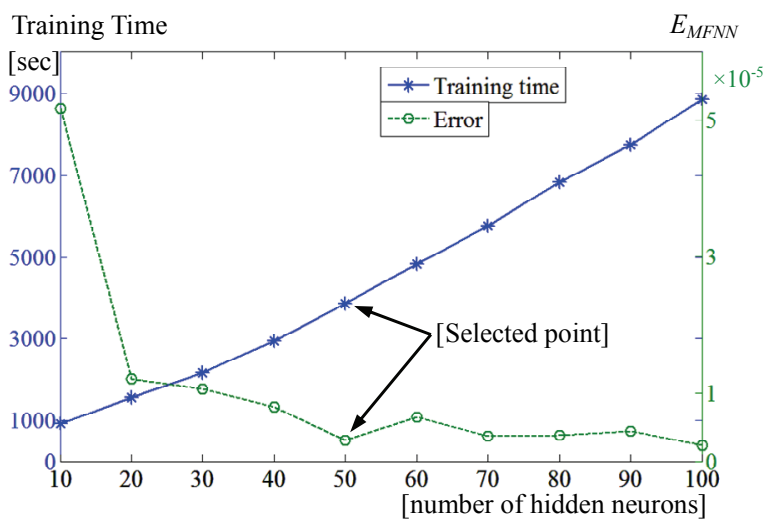

Fig. 5. Training time of MFNN and $E_{M F N N}$ as a function of hidden neuron number

compromise is necessary. In this paper, different numbers of hidden neurons are independently tested. Fig. 5 shows the $E_{M F N N}$ and the corresponding training time for 10 different numbers of hidden neurons, from 10 to 100. From Fig. 5, considering the increase in training time, hidden neurons by more than 50 are redundant to decrease the error. Therefore, in this paper, the number of hidden neurons of the MFNN is selected to be 50 for the nonlinear water wall model, which has $3.00 \times 10^{-6}$ of $E_{M F N N}$ and 3853.6 [sec] of training time.

\subsection{Echo state network model}

The ESN is a special form of RNN with three layers: an input layer, a hidden layer and an output layer. The significant characteristic of the ESN is a hidden layer with a large number of neurons that are sparsely and randomly inter-connected and/or self-connected. This is meant to imitate a biological human brain system. The node is commonly referred to as a "dynamic reservoir" that can be excited by connecting it with the input unit and/or the feedback output unit. A more detailed explanation of the ESN was described by Jaeger [10]. Such ESNs have been recently applied successfully for dynamic system identification and control [11, 19-21].

In this paper, the ESN is used as a second neural network model to describe the nonlinear dynamics of the water wall system. Fig. 6 shows the structure of the ESN used in this paper. In the figure, the ESN has 6 inputs and 3 outputs that are the same as those of a water wall system. The connection weights are divided into 4 categories, $W^{\text {in }}$, $W^{d r}, W^{f b}$ and $W^{o u t}$, which are the input weight matrix, internal weight matrix, output feedback weight matrix and output weight matrix, respectively.

In this figure, $W^{\text {in }}$ is represented with solid lines from the input nodes to the reservoirs; $W^{d r}$ is represented with solid lines among reservoirs; $W^{f b}$ is represented with solid lines from the output nodes to the reservoirs; and $W^{\text {out }}$ is represented with dotted lines from the input, reservoirs and output nodes to the output nodes. When the number

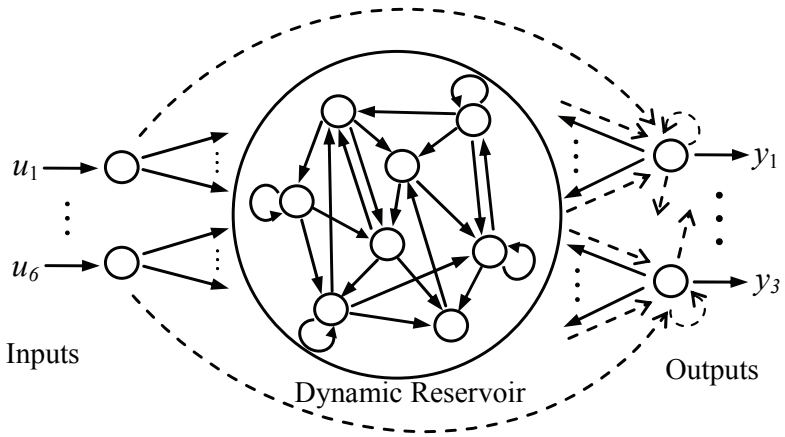

Fig. 6. The architecture of an ESN for a water wall system

of reservoirs is $N_{d r}$, the dimensions of $W^{i n}, W^{d r}, W^{f b}$ and $W^{\text {out }}$ are $\left(N_{d r} \times 6\right),\left(N_{d r} \times N_{d r}\right),\left(N_{d r} \times 3\right)$ and $\left(3 \times\left(N_{d r}+9\right)\right)$, respectively.

The state of the reservoir and output from input in Fig. 6 are computed as follows:

$$
\begin{gathered}
S(n+1)=f\left(W^{\text {in }} U(n)+W^{d r} S(n)+W^{f b} Y(n)\right) \\
Y(n+1)=W^{\text {out }}\left(\begin{array}{c}
U(n) \\
S(n+1) \\
Y(n)
\end{array}\right)
\end{gathered}
$$

where $S(n)=\left[s_{1}(n), \cdots, s_{N d r}(n)\right]^{T}$ is the state of the dynamic reservoir, $U(n)=\left[u_{1}(n), \cdots, u_{6}(n)\right]^{T}$ is the input, $Y(n)=\left[y_{1}(n)\right.$, $\left.y_{2}(n), y_{3}(n)\right]^{T}$ is the output, $f(\cdot)$ is the hyperbolic tangent function and $n$ is a discrete time step.

In the ESN, $W^{i n}, W^{d r}$ and $W^{f b}$ are fixed with random numbers that were initially generated while $W^{\text {out }}$ is trained with the given input-output data. This is a significant difference between ESN and other RNNs, which enable ESN training through a simple linear regression $[10,22]$. Randomly generated $\left(W^{i n}, W^{d r}, W^{f b}\right)$ determine the "echo state properties" which means that "if the network has been run for a long time, the current network state is uniquely determined by the history of the input and the teacher forced output." [12]. A more detailed analysis of the sufficient conditions and necessary conditions for the echo state property are presented by Zhang et al. [23].

To guarantee the echo state properties, $W^{d r}$ is usually generated as follows [16, 19]:

$$
W^{d r}=\alpha \frac{W_{0}^{d r}}{\left|\lambda_{\max }\right|}
$$

where, $W_{0}^{d r}$ is the initial internal weight matrix with a sparse connectivity of $5 \%$ over the range $[-0.5,0.5]$, and its mean value is about zero, $\lambda_{\max }$ is the highest eigenvalue of $W_{0}^{d r}$, and $\alpha$ is a constant of less than one, which is referred to as a spectral radius. Also, $W^{i n}$ and $W^{f b}$ are randomly chosen in the range $[-1,1]$.

The training of the ESN is an off-line calculation of the output weight $W^{\text {out }}$ to minimize the error square as follows: 


$$
\min _{W^{o u t}} \quad E_{E S N}=\sum_{n}^{N}\left(Y_{E S N}(n)-Y(n)\right)^{2}
$$

where $Y_{E S N}$ represents the output of the ESN and $Y$ represents the normalized output of the training data. In (23), initial part of the training data is not considered into the $E_{E S N}$ since the initial dynamic reservoir, $S(0)$, is initially set with an arbitrary value that affects the output. This part is referred to as the "initial washout time" and is usually selected empirically $[10,15,16,19,22]$. In this paper, this time is selected as 500 [sec], and therefore, the number of training data $N$ is 25,000 [step] for ESN.

To minimize the $E_{\mathrm{ESN}}$, the training input $U(n)$, the internal states $S(n+1)$ and the training output $Y(n+1)$ are collected together into a new row of a matrix $M$. That is, $M$ is the concatenated matrix of $U, S$ and $Y$, and its size is $N \times\left(N_{d r}+9\right)$. At the same time, the training outputs $Y(n)$ are collected into another new matrix $T$ with size $(N \times 3)$. Then, the desired $W^{\text {out }}$, which minimize the $E_{\mathrm{ESN}}$, are obtained by multiplying the pseudo-inverse of $M$ with $T$ as follows [15, 19, 22].

$$
W^{\text {out }}=\left\{M^{T}\left(M M^{T}\right)^{-1} T\right\}^{T}=\left(M^{+} T\right)^{T}
$$

where, $M^{+}$denotes the pseudo-inverse of the $M$ matrix. Since training in the ESN is simply performed with (24), the training time for the ESN is mainly determined as the time to calculate the pseudo-inverse. In this paper, the Matlab function $\operatorname{pinv}()$ is used to calculate the pseudoinverse using singular value decomposition. This simple training is a major benefit of the ESN over other neural networks.

In practice, the number of reservoirs $\left(N_{d r}\right)$ and the value of the spectral radius $(\alpha)$ are important parameters for the performance of the ESN. Usually, a large $N_{d r}$ supplies various dynamics to decrease the error while increasing the redundancy for the ESN. A small $\alpha$ decreases the inter-connected weights and the self-connected weights of the dynamic reservoirs. Therefore, a small $\alpha$ might be sufficient to describe the dynamics with a small time constant while a large $\alpha$ is required to describe the

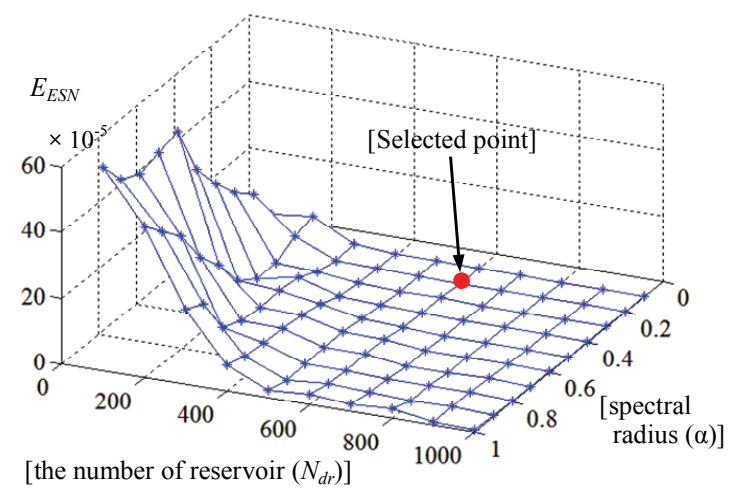

Fig. 7. $E_{E S N}$ as a function of the reservoir number and spectral radius

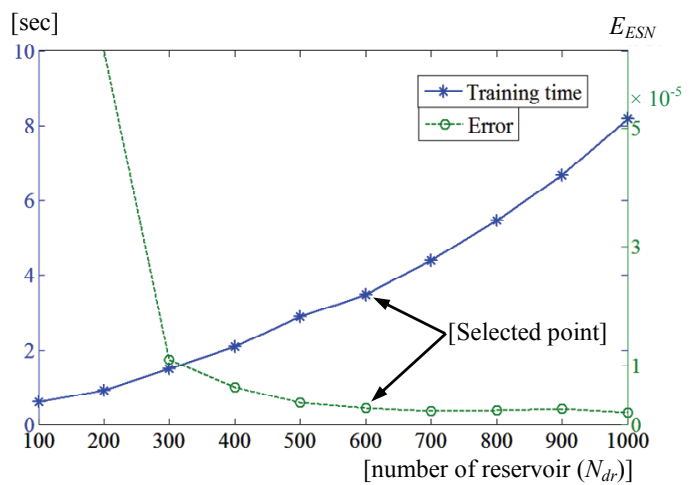

Fig. 8. Training time of the ESN and $E_{E S N}$ as a function of the reservoir number

necessary dynamics with a large time constant [24].

In this paper, different values of $N_{d r}$ and $\alpha$ are tested independently. Fig. 7 shows the $E_{E S N}$ as a function of $N_{d r}$, and $\alpha$ when $N_{d r}$ is $100,200, \ldots, 1000$ and $\alpha$ is $0.1,0.2, \ldots$, 1. Since the $E_{E S N}$ is dependent on a randomly generated initial weight set $\left(W^{i n}, W^{d r}, W^{f b}\right)$, the $E_{E S N}$ of each point in Fig. 7 is selected with the best value of ten initial weight sets. From Fig. 7, the ESN shows a small error with a large $N_{d r}$ and small $\alpha$. From a comparison with the MFNN, an ESN with more than 700 reservoirs and less than 0.2 of $\alpha$ shows a smaller error than that of MFNN. In this paper, the final parameters of the ESN model used to describe the water wall system are selected with 600 for $N_{d r}$ and 0.2 for $\alpha$, which has a $2.73 \times 10^{-6} E_{E S N}$.

Fig. 8 shows the training time of the ESN with respect to $N_{d r}$. Since $\alpha$ does not affect the calculation time with (24), the training time is represented with $N_{d r}$ at 0.2 of $\alpha$ in this figure. Naturally, a large $N_{d r}$ requires a large training time. In Figs. 5 and 8, the training time for the ESN is noted to be of less than 10 [sec] while that of MFNN is of several thousand [sec]. This is one of the important benefits of ESN, which can be trained in a one-shot manner with (24) to minimize (23). The training time for the selected ESN model is of $3.5[\mathrm{sec}]$ in this paper.

\section{Comparison Results}

The performance of the three models, i.e., the linear, MFNN and ESN models, is tested using PRBNS data that were not used during training. To evaluate the performance, the PRBNS input data in Fig. 3 are independently applied to the three models. Then, nonlinear outputs between 3000 [sec] and 4000 [sec] in Fig. 4, which is not used during training, are compared with the three outputs of the MFNN, ESN and linear models.

Fig. 9 shows the comparison of $y_{1}$ for the linear, MFNN and ESN models. Since $y_{1}$ is a linear output, the linear model is identical to the nonlinear model. In this case, both MFNN and ESN show almost the same response as that of the nonlinear model. 


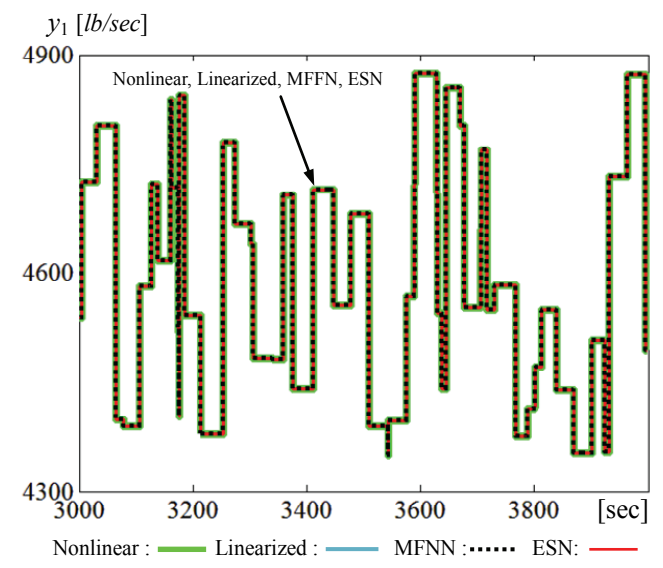

Fig. 9. Comparison of $y_{1}$ for validation data

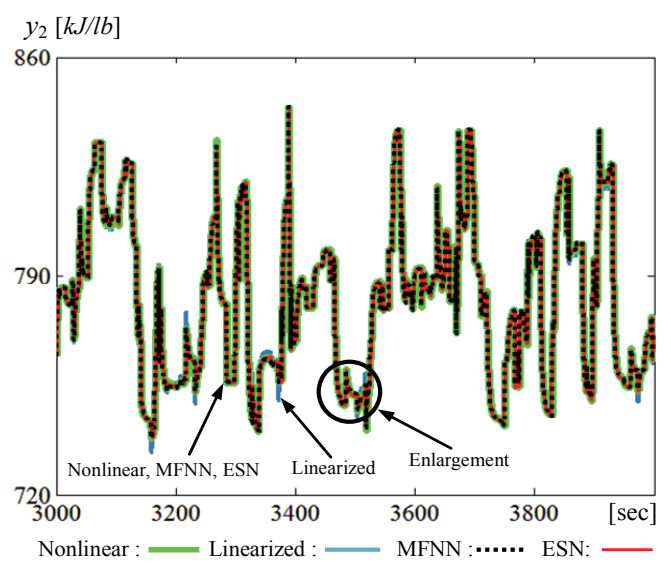

Fig. 10. Comparison of $y_{2}$ for validation data

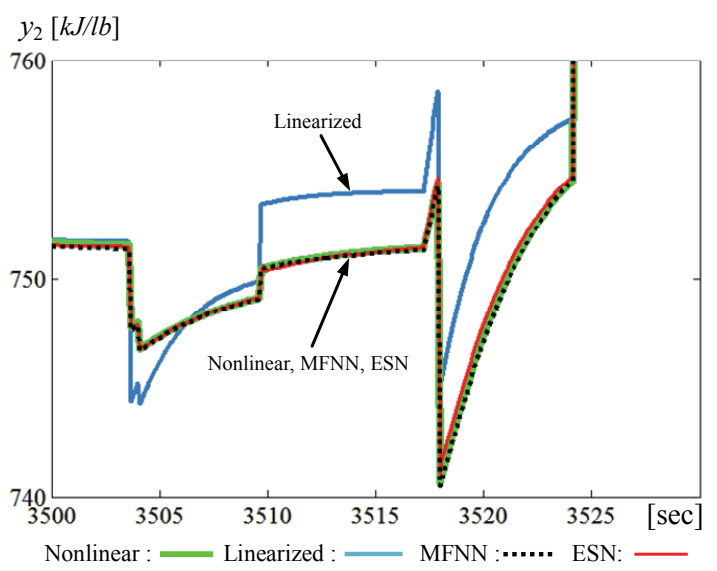

Fig. 11. An enlargement of Fig. 10

Fig. 10 shows the validation results for $y_{2}$, and Fig. 11 is an enlargement of Fig. 10. Since $y_{2}$ is a nonlinear output, the linear model shows some mismatch. This mismatch is expected from the analysis in Section 2.2. In this case, the MFNN and ESN show almost the same results as the nonlinear system. These validation results show that MFNN and ESN have the capability to describe the $y_{2}$ of the nonlinear water wall model properly. Fig. 12 shows the validation results of $y_{3}$, and Fig. 13 is an enlargement of

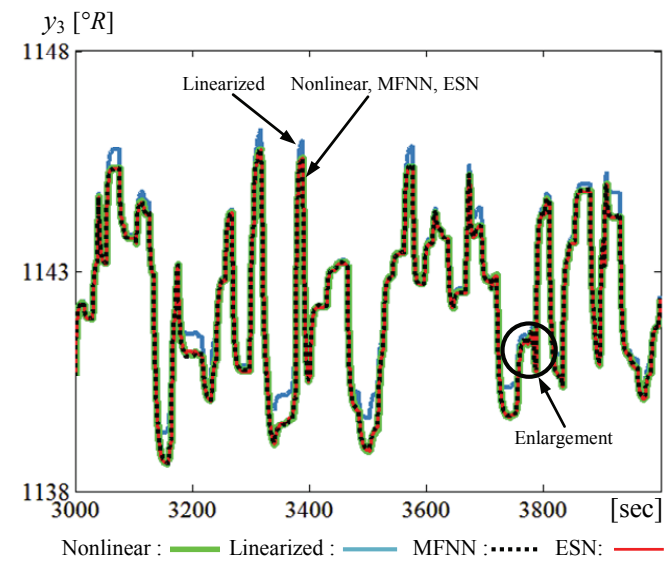

Fig. 12. Comparison of $y_{3}$ for validation data

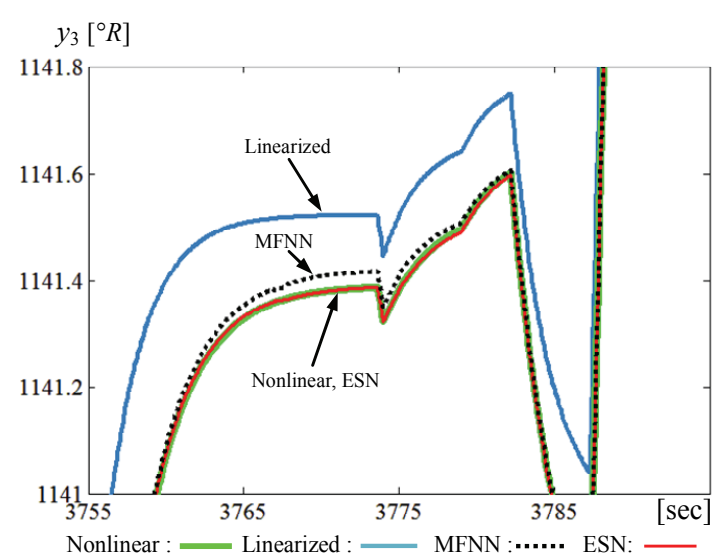

Fig. 13. An enlargement of Fig. 12

Table 2. Comparison of MSE for three models

\begin{tabular}{c|c|c|c}
\hline & Linear Model & MFNN Model & ESN Model \\
\hline$y_{1}$ & 0.0000 & $4.8583 \mathrm{e}-13$ & $2.3554 \mathrm{e}-26$ \\
\hline$y_{2}$ & $1.5637 \mathrm{e}-04$ & $4.1642 \mathrm{e}-06$ & $1.1530 \mathrm{e}-06$ \\
\hline$y_{3}$ & $2.1470 \mathrm{e}-03$ & $1.4864 \mathrm{e}-05$ & $5.3108 \mathrm{e}-06$ \\
\hline
\end{tabular}

Table 3. Comparison of the training time between MFNN and ESN

\begin{tabular}{c|c|c}
\hline & MFNN Model & ESN Model \\
\hline Training time $[\mathrm{sec}]$ & 3853.6 & 3.5 \\
\hline
\end{tabular}

Fig. 12. Since $y_{3}$ is a nonlinear output, the linear model shows some mismatch. The MFNN and ESN show almost the same results as the nonlinear system in the figure. These validation results show that MFNN and ESN have the capability to properly describe the entire dynamics of the nonlinear water wall model.

A quantitative comparison with the mean square error is listed in Table 2. Although both MFNN and ESN exhibit better results than the linear model, ESN shows slightly better results than the MFNN in this case. Table 3 shows the training time for the MFNN model and the ESN model. The simple and fast learning time of ESN is one of the 
most important benefits over other neural network models.

\section{Conclusion}

In this paper, we present a comparison among linear model, multilayer feed-forward neural network (MFNN) model and echo state neural network (ESN) model for a practical water wall system in a 600 [MW] thermal power plant, which is a MIMO nonlinear system.

First, we present an analysis of the results of the linearization of a mathematical water wall model. The change in the pole and steady-state gain is presented as a function of the electric power output. As a result, the system shows quite severe nonlinearity in the design of a linear controller.

Second, we developed an MFNN model and an ESN model without using a mathematical water wall model. Though ESN shows slightly better performance, the results show that both neural network models can provide a satisfactory description of the nonlinear water wall model. Meanwhile, the ESN shows significantly faster training than the MFNN.

From the point of view of control system design, the change in the pole and steady-state gain in this paper can be applied. Though the use of ESN for control and analysis remains to be further developed in future research, we identify the ability of the ESN as a good neural network model for practical nonlinear MIMO identification problems.

\section{Acknowledgements}

This research was supported by the Chung-Ang University Excellent Student Scholarship in 2015 and by Basic Science Research Program through the National Research Foundation of Korea (NRF) funded by the Ministry of Education, Science and Technology (20100025555) and by the Korea Institute for Advancement of Technology (KIAT) grant funded by the Korea Government Ministry of Trade Industry and Energy(MOTIE). (No. N0001075)

\section{References}

[1] C. Liu, H. Wang, J. Ding, and C. Zhen, "An Overview of Modelling and Simulation of Thermal Power Plant," Proc. of the 2011 International Conference on Advanced Systems, pp. 86-91, Zhengzhou, China, 2011.

[2] L. Xueqin, L. Gang, and L. Shangqing, "The development of the boiler Water Wall Tube Inspection," Third International Conference on Electric Utility Deregulation and Restructuring and Poewer Tech- nologies, pp. 2415-2420, Nanjing, April 2008.

[3] P. B. Usoro, "Modeling and Simulation of a Drum Boiler-Turbine Power Plant Under Emergency State Control," Master Degree of Massachusetts Institute of Technology, pp.138-139, May 1977.

[4] U.-C. Moon, and K. Y. Lee, "An Adaptive Dynamic Matrix Control with Fuzzy-Interpolated Step-Response Model for a Drum-Type Boiler-Turbine System," IEEE Transactions on Energy Conversion, Vol. 26, No. 2, pp. 393-401, June 2011.

[5] V. Kecman, Learning and Soft Computing: Support Vector Machines, Neural Networks and Fuzzy Logic Models, Piscataway, NJ: IEEE MIT Press, 2002.

[6] I. Snadburg, J. Lo, C. Fancourt, J. Principe, S. Katagiri, and S. Haykin, Nonlinear Dynamical Systems: Feedforward Neural Network Perspectives, New York: Wiley, 2001.

[7] F. J. Lin, Y. C. Hung, and S. Y. Chen, "FPGA-Based Computed Force Control System Using Elman Neural Network for Linear Ultrasonic Motor," IEEE Transactions on industrial electronics, Vol. 56, No. 4, pp. 1238-1253, April 2009.

[8] Ku, C. C. and K. Y. Lee, "Diagonal Recurrent Neural Network for Dynamic Systems Control," IEEE Transactions on Neural Networks, Vol. 6, pp. 144156, January 1995.

[9] A. F. Atiya and A. G. Parlos, "New results on recurrent network training: Unifying the algorithms and accelerating convergence," IEEE Transactions on Neural Networks, vol. 11, no. 3, pp. 697-709, May 2000.

[10] H. Jaeger, "The echo sate approach to analyzing and training recurrent neural networks-with an Erratum note," Fraunhofer Institute for Autonomous Intelligent Systems (AIS), January 26, 2010.

[11] D. Prokhorov, "Echo state networks: Appeal and challenges," in Proc. IEEE International Conference Neural Networks (IJCNN05), Montreal, PQ, Canada, July 31-August 4, 2005, Vol. 2, pp. 905-910.

[12] H. Jaeger, M. Lukosevicius, D. Popovici and U. Siewert, "Optimization and applications of echo state networks with leaky-integrator neurons," Neural Networks, Vol. 20, special issue, pp. 335-352, 2007.

[13] X. Lin, Z. Yang, and Y. Song, "Intelligent stock trading system based on improved technical analysis and Echo State Network," Expert Systems with Applications, Vol. 38, pp. 11347-11354, 2011.

[14] K. Ishii, T. van der Zant, V. Becanovic and P. Ploger, "Optimization of Parameter of Echo State Network and Its Application to Underwater Robot," SICE Annual Conference in Sapporo, pp. 2800-2805, August 2004.

[15] J. Dai, P. Zhang, J. Mazumdar, R. G. Harley and G. K. Venayagamoorthy "A Comparison of MLP, RNN and ESN in Determining Harmonic Contribution from Nonlinear Loads," $34^{\text {th }}$ Annual Conference of IEEE 
on Industrial Electronics, pp. 3025-3032, November 2008.

[16] Y. Pan, and J. Wang, "Model Predictive Control of Unknown Nonlinear Dynamical Systems Based on Recurrent Neural Networks," IEEE Transactions on Industrial Electronics, Vol. 59, No. 8, pp. 3089-3101, August 2012.

[17] R. Johansson, System Modeling and Identification, Englewood Cliffs, NJ, USA: Prentice-Hall, 1992.

[18] S. Haykin, Neural Networks, Ottawa, On, Canada: Maxwell Macmillan, 1994.

[19] J. Mazumdar, and R. G. Harley, "Utilization of echo state networks for differentiating source and nonlinear load harmonics in the utility network," IEEE Transactions on power Electronics, Vol. 23, No. 6, pp. 2738-2745, November 2008.

[20] G. Venayagamoorthy, "Online design of an echo state network based wide area monitor for a multimachine power system," Neural Networks, Vol. 20, No. 3, pp. 404-413, April 2007.

[21] D. Li, M. Han, and J. Wang, "Chaotic time series prediction based on a novel robust echo state network," IEEE Transactions on Neural Networks and Learning Systems, vol. 23, no. 5, pp. 787-799, May 2012.

[22] H. Jaeger, "A tutorial on training recurrent neural networks, covering BPPT, RTRL, EKF and echo state network approach," GMD-Report 159, German National Research Center for Information Technology, 2002.

[23] B. Zhang, D. J. Miller, and Y. Wang, "Nonlinear system modeling with random matrices: Echo state networks revisited," IEEE Transactions on Neural Networks and Learning systems, Vol. 23, No. 1, pp. 175-182, January 2012.

[24] A. Millea, "Explorations in echo state networks," Department of Artificial Intelligence, Groningen University, MA, Netherlands, S2064235, June 2014.

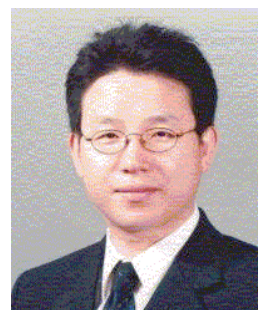

Un-Chul Moon received his B.S., M.S. and Ph.D. degrees from Seoul National University, Korea, in 1991, 1993 and 1996, respectively, all in Electrical Engineering. From 2000, he was with the Woo-Seok University, Korea, and from 2002 with the Chung-Ang University, Korea, where he is currently an Associate Professor of Electrical Engineering. His current research interests are in the areas of power system analysis, computational intelligence and automation.

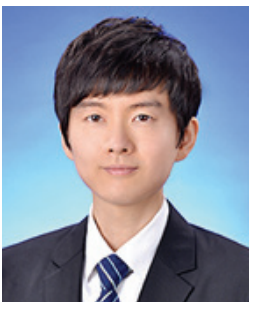

Jaewoo Lim received his B.S. degree in Electrical and Electronics Engineering from the Chung-Ang University, Seoul, Korea, in 2014. He is currently working toward the M.S degree in Chung-Ang University of Electrical and Electronics Engineering. His research interests include control and modeling of fossil power plant and power system analysis.

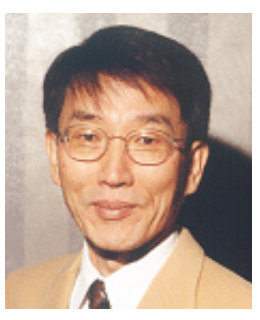

Kwang Y. Kee (F'01) received the B.S. degree in Electrical Engineering from Seoul National University, Seoul, Korea, in 1964, the M.S. degree in Electrical Engineering from North Dakota State University, Fargo, in 1968, and the $\mathrm{Ph} . \mathrm{D}$. degree in System Science from Michigan State University, East Lansing, in 1971. He has been on the faculties of Michigan State, Oregon State, University of Houston, Penn State, and Baylor University, where he is a Professor and Chair of Electrical and Computer Engineering. His interests are power system control, operation and planning and intelligent system techniques, and their application to power system and power plant control. Dr. Lee is an Editor of IEEE Transactions on Energy Conversion and former Associate Editor of IEEE Transactions on Neural Networks. Dr. Lee is a Fellow of the IEEE. 